\title{
Probing the cosmographic parameters to distinguish between dark energy and modified gravity models (Research Note)
}

\author{
F. Y. Wang ${ }^{1,2}$, Z. G. Dai ${ }^{1}$, and Shi $\mathrm{Qi}^{3,4}$ \\ 1 Department of Astronomy, Nanjing University, Nanjing 210093, PR China \\ e-mail: fayinwang@nju.edu.cn \\ 2 Department of Astronomy, University of Texas at Austin, Austin, TX 78712, USA \\ Purple Mountain Observatory, Chinese Academy of Sciences, Nanjing 210008, PR China \\ 4 Joint Center for Particle, Nuclear Physics and Cosmology, Nanjing University - Purple Mountain Observatory, \\ Nanjing 210093, PR China
}

Received 6 March 2009 / Accepted 4 August 2009

ABSTRACT

\begin{abstract}
Aims. In this paper we investigate the deceleration, jerk and snap parameters to distinguish between the dark energy and modified gravity models using high redshift gamma-ray bursts (GRBs) and supernovae (SNe).

Methods. We first derive the expressions of deceleration, jerk and snap parameters in dark energy and modified gravity models. In order to constrain the cosmographic parameters, we calibrate the GRB luminosity relations without assuming any cosmological models using SNe Ia. Then we constrain the model parameters (including dark energy and modified gravity models) using type Ia supernovae and gamma-ray bursts. Finally we calculate the cosmographic parameters. GRBs can extend the redshift-distance relation up to high redshifts, because they can be detected to high redshifts.

Results. We find that the statefinder pair $(r, s)$ could not be used to distinguish between some dark energy and modified gravity models, but these models could be differentiated by the snap parameter. Using the model-independent constraints on cosmographic parameters, we conclude that the $\Lambda \mathrm{CDM}$ model is consistent with current data.
\end{abstract}

Key words. gamma rays: bursts - cosmology: cosmological parameters - cosmology: distance scale

\section{Introduction}

Recent observations of the Hubble relation of distant type Ia supernovae (SNe Ia) have provided strong evidence for acceleration of the present universe (Riess et al. 1998; Perlmutter et al. 1999). The observations of the spectrum of cosmic microwave background (CMB) anisotropies (Spergel et al. 2003, 2007), large-scale structure (LSS) (Tegmark et al. 2004; Eisenstein et al. 2005) and the distance-redshift relation to X-ray galaxy clusters (Allen et al. 2004, 2007) also confirm that the universe is accelerating. Possible explanations for this acceleration have been proposed. A negative pressure term called dark energy is taken into account, such as in the cosmological constant model with equation of state $w=p / \rho=-1$ (Weinberg 1989), an evolving scalar field (Peeble \& Ratra 1988; Caldwell et al. 1998), phantom energy for which the sum of the pressure and energy density is negative, and the Chaplygin gas (Kamenshchik et al. 2001). All the above models for acceleration are obtained by introducing a new energy component called dark energy. Alternative models, in which gravity is modified, can also drive the universe acceleration, e.g., the Dvali-Gabadadze-Porrati (DGP) model (Dvali et al. 2000; Deffayet et al. 2002), Cardassian expansion model (Freese \& Lewis 2002; Wang et al. 2003), and the $f(R)$ gravity model (Vollick 2003; Carroll et al. 2004).

These two families of models, dark energy and modified gravity, are fundamentally different. An important question is whether it is possible to distinguish between the modified gravity and dark energy models that have nearly the same cosmic expansion history. Much work has been done on this topic. A usually-discussed quantity is the growth rate of cosmological density perturbations, which should be different in the models depending on different gravity theory even if they have an identical cosmic expansion history. Recently, there have been extensive discussions on discriminating dark energy and modified gravity models using the matter density perturbation growth factor (Linder 2005). But Kunz \& Sapone (2007) demonstrated that the growth factor is not sufficient to distinguish between modified gravity and dark energy (Kunz \& Sapone 2007). They found that a generalized dark energy model can match the growth rate of the Dvali-Gabadadze-Porrati model and reproduce the $3+1$ dimensional metric perturbations.

On the other hand, the statefinder pair $(r, s)$ has also been proposed to distinguish between the models, where $r \equiv \dot{a} / a H^{3}$ and $s \equiv(r-1) / 3(q-1 / 2)$. Sahni et al. (2003) demonstrated that the statefinder diagnostic could effectively discriminate different forms of dark energy (Sahni et al. 2003). Alam et al. (2003) investigated the cosmological constant, quintessence, Chaplygin gas, and braneworld models using the statefinder diagnostic, and found that the statefinder pair could differentiate between these models (Alam et al. 2003). Different cosmological models exhibit qualitatively different trajectories of evolution in the $r-s$ plane. The statefinder diagnostic has been extensively used in many models (Gorini et al. 2003). But the statefinder pair is difficult to measure with cosmological observations (Visser 2004; Cattoën \& Visser 2007).

The present values of cosmographic parameters can be determined from observations (Riess et al. 2004; Visser 2004). Caldwell \& Kamionkowski (2004) showed that the jerk 
parameter could probe the spatial curvature of the universe (Caldwell \& Kamionkowski 2004). The deceleration, jerk and snap parameters are related to the second, third and fourth derivative of the scale factor respectively. Visser (2004) expanded the Hubble law to fourth order in redshift including the snap parameter and put constraints on the deceleration and jerk parameters using SNe Ia (Visser 2004). Rapetti et al. (2007) constrained the deceleration and jerk parameters from $\mathrm{SNe}$ Ia and X-ray cluster gas mass fraction measurements. For a redshift range of $\mathrm{SNe}$ Ia, the terms beyond the cubic power of the Hubble law can be neglected. In order to put a narrow constraint on the snap parameter, we need high-redshift objects. GRBs may be a useful tool. GRBs can be detectable out to very high redshifts (Ciardi \& Loeb 2000). The farthest burst detected so far is GRB 090423, which is at $z=8.2$ (Olivares et al. 2009). A lot of work in this so-called GRB cosmology has been published (Dai et al. 2004; Ghirlanda et al. 2004; Di Girolamo et al. 2005; Firmani et al. 2005; Friedman \& Bloom 2005; Lamb et al. 2005; Liang \& Zhang 2005, 2006; Xu et al. 2005; Wang \& Dai 2006; Schaefer 2007; Wright 2007; Wang et al. 2007; Gong \& Chen 2007; Li et al. 2008; Liang et al. 2008; Qi et al. 2008a,b; Basilakos \& Perivolaropoulos 2008; Kodama et al. 2008; Wang et al. 2009). Very recently, Schaefer (2007) used 69 GRBs and five relations to build the Hubble diagram out to $z=6.60$ and discussed the properties of dark energy in several dark energy models (Schaefer 2007). He found that the GRB Hubble diagram is consistent with the concordance cosmology. Liang et al. (2008) calibrated the luminosity relations of GRBs by interpolating from the Hubble diagram of SNe Ia at $z<1.4$ with the assumption that objects at the same redshift should have the same luminosity distance (Liang et al. 2008). This method is modelindependent. More recently, Capozziello \& Izzo (2008) used the Liang et al. (2008) results to constrain the cosmographic parameters and found that the results calibrated by SNe Ia data agree with the $\Lambda$ CDM model. Cardone et al. (2009) used 83 GRBs and six correlations to build the Hubble diagram.

Riess et al. (2004) found that the jerk $j_{0}$ is positive at the $92 \%$ confidence level based on their "gold" dataset and is positive at the $95 \%$ confidence level based on their "gold+silver" dataset. Neither explicit upper bounds are given for the jerk nor are any constraints placed on the snap $s_{0}$. Rapetti et al. (2007) measured $q_{0}=-0.81 \pm 0.14$ and $j=2.16_{-0.75}^{+0.81}$ in a flat model with constant jerk (Rapetti et al. 2007). Capozziello \& Izzo (2008) used 27 GRBs to derive the values of the cosmographic parameters. They found $q_{0}=-0.78 \pm 0.20, j_{0}=0.62 \pm 0.86$ and $s_{0}=8.32 \pm 12.16$. In this paper, we use more GRB data to constrain the cosmography parameters in several dark energy and modified gravity models.

We calibrate the luminosity relations of GRBs using SNe Ia and calculate the deceleration, jerk and snap parameters of several dark energy and modified gravity models using SNe Ia and GRBs. We also use a model-independent method to constrain the cosmographic parameters. We find that in some models the jerk parameters are almost equal to each other. So this parameter is not used to distinguish between the models. However, the snap parameters in all the models are different, so we can distinguish between the models using the snap parameter.

The structure of this paper is as follows. In Sect. 2 we introduce the Hubble, deceleration, jerk and snap parameters. In Sect. 3 we derive expressions of cosmographic parameters of the Hubble law in several dark energy models. In Sect. 4 we present expressions of cosmographic parameters of the Hubble law in modified gravity models. The constraints on model parameters and cosmographic parameters of the Hubble law are given in Sect. 5. Finally, Sect. 6 contains conclusions and discussions.

\section{Hubble, deceleration, jerk and snap parameters}

The expansion rate of the Universe can be written in terms of the Hubble parameter, $H=\dot{a} / a$, where $a$ is the scale factor and $\dot{a}$ is its first derivative with respect to time. As we known that $q$ is the deceleration parameter, related to the second derivative of the scale factor, $j$ is the so-called "jerk" or statefinder parameter, related to the third derivative of the scale factor, and $s$ is the socalled "snap" parameter, which is related to the fourth derivative of the scale factor. These quantities are defined as

$q=-\frac{1}{H^{2}} \frac{\ddot{a}}{a}$

$j=\frac{1}{H^{3}} \frac{\dot{a}}{a}$

$s=\frac{1}{H^{4}} \frac{\ddot{a}}{a}$

The deceleration, jerk and snap parameters are dimensionless, and a Taylor expansion of the scale factor around $t_{0}$ provides

$$
\begin{aligned}
a(t)= & a_{0}\left\{1+H_{0}\left(t-t_{0}\right)-\frac{1}{2} q_{0} H_{0}^{2}\left(t-t_{0}\right)^{2}+\frac{1}{3 !} j_{0} H_{0}^{3}\left(t-t_{0}\right)^{3}\right. \\
& \left.+\frac{1}{4 !} s_{0} H_{0}^{4}\left(t-t_{0}\right)^{4}+O\left[\left(t-t_{0}\right)^{5}\right]\right\}
\end{aligned}
$$

and so the luminosity distance

$$
\begin{aligned}
d_{\mathrm{L}}= & \frac{c}{H_{0}}\left\{z+\frac{1}{2}\left(1-q_{0}\right) z^{2}-\frac{1}{6}\left(1-q_{0}-3 q_{0}^{2}+j_{0}\right) z^{3}\right. \\
& +\frac{1}{24}\left[2-2 q_{0}-15 q_{0}^{2}-15 q_{0}^{3}+5 j_{0}+10 q_{0} j_{0}+s_{0}\right] z^{4} \\
& \left.+O\left(z^{5}\right)\right\}
\end{aligned}
$$

(Visser 2004). For the redshift range of SNe Ia the terms beyond the cubic power in Eq. (5) can be neglected. If models have the same deceleration and jerk parameters, we can see degeneracy of these models from Eq. (5). Therefore we must measure the snap parameters to distinguish between the models. This needs highredshift objects. The relations among the $q(z), j(z)$ and $s(z)$ are

$j(z)=q(z)+2 q^{2}(z)+(1+z) \frac{\mathrm{d} q}{\mathrm{~d} z}(z) ;$

$s(z)=-(1+z) \frac{\mathrm{d} j}{\mathrm{~d} z}(z)-2 j(z)-3 j(z) q(z)$.

The Friedmann equation is

$H^{2}=\left(\frac{\dot{a}}{a}\right)^{2}=\frac{8 \pi G}{3} \sum_{i} \rho_{i}$.

From Einstein's equations, we can obtain the dynamical equation of the universe

$\frac{\ddot{a}}{a}=-\frac{4 \pi G}{3} \sum_{i}\left(\rho_{i}+3 P_{i}\right)$.

The conservation equation is

$\dot{\rho}_{i}+3 H\left(\rho_{i}+P_{i}\right)=0$. 
In order to derive the jerk and snap parameter, we differentiate Eq. (9)

$$
\begin{aligned}
\dot{a}= & -\frac{4 \pi G}{3} \sum_{i}\left[\dot{a} \rho_{i}\left(1+3 w_{i}\right)+a \dot{\rho}_{i}\left(1+3 w_{i}\right)+a \rho_{i} \times 3 \dot{w}_{i}\right] \\
= & -\frac{4 \pi G}{3} \sum_{i}\left[a H \rho_{i}\left(1+3 w_{i}\right)-3 H a \rho_{i}\left(1+w_{i}\right)\left(1+3 w_{i}\right)\right. \\
& \left.+a \rho_{i} \times 3 \dot{w}_{i}\right], \\
\ddot{a}= & -\frac{4 \pi G}{3} \sum_{i} \frac{\mathrm{d}\left[\dot{a} \rho_{i}\left(1+3 w_{i}\right)+a \dot{\rho}_{i}\left(1+3 w_{i}\right)+a \rho_{i} \times 3 \dot{w}_{i}\right]}{\mathrm{d} t} \\
= & -\frac{4 \pi G}{3} \sum_{i}\left[\ddot{a} \rho_{i}\left(1+3 w_{i}\right)+2 \dot{a} \dot{\rho}_{i}\left(1+3 w_{i}\right)+6 \dot{a} \rho_{i} \dot{w}_{i}\right. \\
& \left.+a \ddot{\rho}_{i}\left(1+3 w_{i}\right)+6 a \dot{\rho}_{i} \dot{w}_{i}+a \rho_{i} \times 3 \ddot{w}_{i}\right] .
\end{aligned}
$$

\section{Dark energy models}

\section{1. $w(z)$ parameterization model}

We first consider the dark energy with a constant equation of state

$w(z)=w_{0}$.

For this model, we obtain

$q_{0}^{\mathrm{XCDM}}=\frac{3}{2}\left[1+w_{0}\left(1-\Omega_{\mathrm{M}}\right)\right]-1$,

$\left.\frac{\mathrm{d} q}{\mathrm{~d} z}\right|_{0} ^{\mathrm{XCDM}}=\frac{9}{2} w_{0}^{2}\left(1-\Omega_{\mathrm{M}}\right) \Omega_{\mathrm{M}}$,

$\left.\frac{\mathrm{d} j}{\mathrm{~d} z}\right|_{0} ^{\mathrm{XCDM}}=-\frac{27}{2} w_{0}^{2}\left(1+w_{0}\right)\left(\Omega_{\mathrm{M}}-1\right) \Omega_{\mathrm{M}}$,

$j_{0}^{\mathrm{XCDM}}=\frac{1}{2}\left(2+9\left(1-\Omega_{\mathrm{M}}\right) w_{0}+9\left(1-\Omega_{\mathrm{M}}\right) w_{0}^{2}\right)$,

$$
\begin{aligned}
s_{0}^{\mathrm{XCDM}}= & \frac{1}{4}\left(-14-81\left(1-\Omega_{\mathrm{M}}\right) w_{0}-9\left(16-19 \Omega_{\mathrm{M}}+3 \Omega_{\mathrm{M}}^{2}\right) w_{0}^{2}\right. \\
& \left.-27\left(3-4 \Omega_{\mathrm{M}}+\Omega_{\mathrm{M}}^{2}\right) w_{0}^{3}\right) .
\end{aligned}
$$

These expressions are consistent with Bertolami \& Silva (2006).

A more interesting approach to explore dark energy is to use a time-dependent dark energy model. The simplest parameterization including two parameters is (Maor et al. 2001; Weller \& Albrecht 2001)

$w(z)=w_{0}+w_{1} z$

In this dark energy model the luminosity distance is (Linder 2003)

$$
\begin{aligned}
d_{\mathrm{L}}= & c H_{0}^{-1}(1+z) \int_{0}^{z} \mathrm{~d} z\left[(1+z)^{3} \Omega_{\mathrm{M}}\right. \\
& \left.+\left(1-\Omega_{\mathrm{M}}\right)(1+z)^{3\left(1+w_{0}-w_{1}\right)} \mathrm{e}^{3 w_{1} z}\right]^{-1 / 2} .
\end{aligned}
$$

The cosmographic parameters are:

$q_{0}^{\mathrm{WCDM}}=\frac{1}{2}+\frac{3}{2}\left(1-\Omega_{\mathrm{M}}\right) w_{0}$,

$$
\begin{aligned}
j_{0}^{\mathrm{WCDM}}= & \frac{1}{2}\left(2+9\left(1-\Omega_{\mathrm{M}}\right) w_{0}+9\left(1-\Omega_{\mathrm{M}}\right) w_{0}^{2}\right. \\
& \left.+3\left(1-\Omega_{\mathrm{M}}\right) w_{1}\right) \\
s_{0}^{\mathrm{WCDM}}= & \frac{1}{4}\left(-14-9\left(16-19 \Omega_{\mathrm{M}}+3 \Omega_{\mathrm{M}}^{2}\right) w_{0}^{2}\right. \\
& -27\left(3-4 \Omega_{\mathrm{M}}+\Omega_{\mathrm{M}}^{2}\right) w_{0}^{3}-45\left(1-\Omega_{\mathrm{M}}\right) w_{1} \\
& \left.+9 w_{0}\left(1-\Omega_{\mathrm{M}}\right)\left(-9-\left(\Omega_{\mathrm{M}}-7\right) w_{1}\right)\right) .
\end{aligned}
$$

We consider the Chevallier-Polarski-Linder parameterization (Chevallier \& Polarski 2001; Linder 2003)

$w(z)=w_{0}+\frac{w_{1} z}{1+z}$.

The luminosity distance is (Chevallier \& Polarski 2001; Linder 2003)

$$
\begin{aligned}
d_{\mathrm{L}}= & c H_{0}^{-1}(1+z) \int_{0}^{z} \mathrm{~d} z\left[(1+z)^{3} \Omega_{\mathrm{M}}\right. \\
& \left.+\left(1-\Omega_{\mathrm{M}}\right)(1+z)^{3\left(1+w_{0}+w_{1}\right)} \mathrm{e}^{-3 w_{1} z /(1+z)}\right]^{-1 / 2} .
\end{aligned}
$$

The cosmographic parameters are:

$q_{0}^{\mathrm{CPL}}=\frac{1}{2}+\frac{3}{2}\left(1-\Omega_{\mathrm{M}}\right) w_{0}$

$$
\begin{aligned}
j_{0}^{\mathrm{CPL}}= & \frac{1}{2}\left(2+9\left(1-\Omega_{\mathrm{M}}\right) w_{0}+9\left(1-\Omega_{\mathrm{M}}\right) w_{0}^{2}\right. \\
& \left.+3\left(1-\Omega_{\mathrm{M}}\right) w_{1}\right)
\end{aligned}
$$

$$
\begin{aligned}
s_{0}^{\mathrm{CPL}}= & \frac{1}{4}\left(-14-9\left(16-19 \Omega_{\mathrm{M}}+3 \Omega_{\mathrm{M}}^{2}\right) w_{0}^{2}\right. \\
& -27\left(3-4 \Omega_{\mathrm{M}}+\Omega_{\mathrm{M}}^{2}\right) w_{0}^{3}-33\left(1-\Omega_{\mathrm{M}}\right) w_{1} \\
& \left.+9 w_{0}\left(1-\Omega_{\mathrm{M}}\right)\left(-9-\left(\Omega_{\mathrm{M}}-7\right) w_{1}\right)\right) .
\end{aligned}
$$

Capozziello et al. (2008) and Capozziello \& Izzo (2008) also derived cosmographic parameters in this model. Our results are consistent with theirs.

\subsection{Generalized Chaplygin gas model}

We consider the generalized Chaplygin gas (GCG) model, which is characterized by the equation of state

$p_{\mathrm{GCG}}=-A / \rho_{\mathrm{GCG}}^{\alpha}$.

We can integrate the conservation equation for generalized Chaplygin gas, leading to

$\rho_{\mathrm{GCG}}=\rho_{\mathrm{GCG} 0}\left[A_{s}+\left(1-A_{s}\right) a^{-3(1+\alpha)}\right]^{1 /(1+\alpha)}$

where $\rho_{\mathrm{Ch} 0}$ is the energy density of GCG today, and $A_{s}=$ $A / \rho_{\text {Ch0 } 0}^{1+\alpha}$. An attractive feature of the model is that it can unify dark energy and dark matter. The reason is that, from Eq. (30), the GCG behaves like dustlike matter at an early epoch and as a cosmological constant at a later epoch (Kamenshchik et al. 2001; Bento et al. 2002). The Friedmann equation can be expressed as

$H^{2}\left(z, H_{0}, A_{s}, \alpha\right)=H_{0}^{2} E^{2}\left(z, A_{s}, \alpha\right)$, 
where

$$
\begin{aligned}
E^{2}\left(z, A_{s}, \alpha\right)= & \Omega_{\mathrm{b}}(1+z)^{3}+\left(1-\Omega_{\mathrm{b}}\right) \\
& \times\left[A_{s}+\left(1-A_{s}\right)(1+z)^{3(1+\alpha)}\right]^{\frac{1}{1+\alpha}},
\end{aligned}
$$

$\Omega_{\mathrm{b}}$ is the density parameter of the baryonic matter. The luminosity distance is

$$
\begin{aligned}
d_{\mathrm{L}}= & c H_{0}^{-1}(1+z) \int_{0}^{z} \mathrm{~d} z\left\{(1+z)^{3} \Omega_{\mathrm{b}}+\left(1-\Omega_{\mathrm{b}}\right)\right. \\
& \left.\times\left[A_{s}+\left(1-A_{s}\right)(1+z)^{3(1+\alpha)}\right]^{\frac{1}{1+\alpha}}\right\}^{-1 / 2} .
\end{aligned}
$$

For the GCG model we obtain (Bertolami \& Silva 2006; Wang et al. 2009)

$$
\begin{aligned}
& q_{0}^{\mathrm{GCG}}= \frac{3}{2}\left(1-A_{s}\right)-1 \\
&\left.\frac{\mathrm{d} q}{\mathrm{~d} z}\right|_{0} ^{\mathrm{GCG}}=\frac{9}{2} A_{s}\left(1-A_{s}\right)(1+\alpha), \\
&\left.\frac{\mathrm{d} j}{\mathrm{~d} z}\right|_{0} ^{\mathrm{GCG}}=-\frac{27}{2} \alpha(1+\alpha)\left(2 A_{s}-1\right)\left(A_{s}-1\right) A_{s}, \\
& j_{0}^{\mathrm{GCG}}=\frac{3}{4}\left(1-A_{s}\right)\left(1+(3+6 \alpha) A_{s}\right), \\
& s_{0}^{\mathrm{GCG}}=\frac{3}{8}\left(A_{s}-1\right)\left(7+6\left(2+\alpha-6 \alpha^{2}\right) A_{s}\right. \\
&\left.\quad+9\left(-3+2 \alpha+8 \alpha^{2}\right) A_{s}^{2}\right) .
\end{aligned}
$$

\section{Modified gravity models}

\subsection{Cardassian expansion model}

The original Cardassian model was first introduced in Freese \& Lewis (2002) as a possible alternative to explain the acceleration of the universe. They modified the Friedmann equation as

$H^{2}=\frac{8 \pi G}{3} \rho_{\mathrm{m}}+B \rho_{\mathrm{m}}^{n}$.

This model has no energy component besides ordinary matter. If we consider a spatially flat FRW universe, the Friedmann equation is modified as Eq. (39). The universe undergoes acceleration requiring $n<2 / 3$. If $n=0$, it is the same as the cosmological constant universe. We can obtain $H(z)$ by using Eq. (39) and $\rho_{\mathrm{m}}=\rho_{\mathrm{m}}(1+z)^{3}=\Omega_{\mathrm{m}} \rho_{\mathrm{c}}(1+z)^{3}$,

$H(z)^{2}=H_{0}^{2}\left[\Omega_{\mathrm{m}}(1+z)^{3}+\left(1-\Omega_{\mathrm{m}}\right)(1+z)^{3 n}\right]$,

where $\rho_{\mathrm{c}}=3 H_{0}^{2} / 8 \pi G$ is the critical density of the universe. The luminosity distance in this model is

$d_{\mathrm{L}}=c H_{0}^{-1}(1+z) \int_{0}^{z} \mathrm{~d} z\left[(1+z)^{3} \Omega_{\mathrm{m}}+\left(1-\Omega_{\mathrm{m}}\right)(1+z)^{3 n}\right]^{-1 / 2}$.

For the Cardassian expansion model, we obtain

$q_{0}^{\text {Card }}=\frac{1}{2}+\frac{3}{2}(1-n)\left(\Omega_{\mathrm{M}}-1\right)$,

$\left.\frac{\mathrm{d} q}{\mathrm{~d} z}\right|_{0} ^{\mathrm{Card}}=\frac{9}{2}(n-1)^{2}\left(1-\Omega_{\mathrm{M}}\right) \Omega_{\mathrm{M}}$

$$
\begin{aligned}
\left.\frac{\mathrm{d} j}{\mathrm{~d} z}\right|_{0} ^{\text {Card }}= & \frac{27}{2}(n-1)^{2}\left(1-\Omega_{\mathrm{M}}\right) \Omega_{\mathrm{M}} n \\
j_{0}^{\text {Card }}= & \frac{1}{2}\left(2+9 n\left(\Omega_{\mathrm{M}}-1\right)+9 n^{2}\left(1-\Omega_{\mathrm{M}}\right)\right) \\
s_{0}^{\text {Card }}= & \frac{1}{4}\left(4-18 \Omega_{\mathrm{M}}-27 n^{3}\left(3-4 \Omega_{\mathrm{M}}+\Omega_{\mathrm{M}}^{2}\right)\right. \\
& \left.-9 n\left(4-7 \Omega_{\mathrm{M}}+3 \Omega_{\mathrm{M}}^{2}\right)+9 n^{2}\left(11-17 \Omega_{\mathrm{M}}+6 \Omega_{\mathrm{M}}^{2}\right)\right) .
\end{aligned}
$$

\subsection{Dvali-Gabadadze-Porrati model}

In the DGP model the modified Friedmann equation due to the presence of an infinite-volume extra dimension is (Deffayet et al. 2002)

$H^{2}=H_{0}^{2}\left[\Omega_{k}(1+z)^{2}+\left(\sqrt{\Omega_{r_{\mathrm{c}}}}+\sqrt{\Omega_{r_{\mathrm{c}}}+\Omega_{\mathrm{m}}(1+z)^{3}}\right)^{2}\right]$,

where the bulk-induced term, $\Omega_{r_{\mathrm{c}}}$, is defined as

$\Omega_{r_{\mathrm{c}}} \equiv 1 / 4 r_{\mathrm{c}}^{2} H_{0}^{2}$.

For a flat universe, $\Omega_{k}=0$. In the above equation, $r_{\mathrm{c}}$ is the crossover scale beyond which the gravitational force follows the 5-dimensional $1 / r^{3}$ behavior. Note that on short length scales $r \ll r_{\mathrm{c}}$ (at early times) the gravitational force follows the usual four-dimensional $1 / r^{2}$ behavior. For a spatially flat universe, $\Omega_{r_{\mathrm{c}}}=\left(1-\Omega_{\mathrm{m}}\right)^{2} / 4$. We obtain

$q_{0}^{\mathrm{DGP}}=\frac{1}{2}+\frac{3}{2} \frac{\Omega_{\mathrm{M}}-1}{1+\Omega_{\mathrm{M}}}$,

$\left.\frac{\mathrm{d} q}{\mathrm{~d} z}\right|_{0} ^{\mathrm{DGP}}=\frac{9 \Omega_{\mathrm{M}}\left(1-\Omega_{\mathrm{M}}\right)}{\left(1+\Omega_{\mathrm{M}}\right)^{3}}$

$\left.\frac{\mathrm{d} j}{\mathrm{~d} z}\right|_{0} ^{\text {DGP }}=\frac{54 \Omega_{\mathrm{M}}^{3}\left(1-\Omega_{\mathrm{M}}\right)}{\left(1+\Omega_{\mathrm{M}}\right)^{5}}$,

$j_{0}^{\text {DGP }}=\frac{1+3 \Omega_{\mathrm{M}}-6 \Omega_{\mathrm{M}}^{2}+10 \Omega_{\mathrm{M}}^{3}}{\left(1+\Omega_{\mathrm{M}}\right)^{3}}$,

$s_{0}^{\mathrm{DGP}}=\frac{1-4 \Omega_{\mathrm{M}}-35 \Omega_{\mathrm{M}}^{2}-26 \Omega_{\mathrm{M}}^{3}+32 \Omega_{\mathrm{M}}^{4}-80 \Omega_{\mathrm{M}}^{5}}{\left(1+\Omega_{\mathrm{M}}\right)^{5}}$.

\section{3. $f(R)$ gravity}

$f(R)$ gravity models, in which the gravitational Lagrangian is a function of the curvature scalar $R$, also can explain the current cosmic acceleration (Vollick 2003; Carroll et al. 2004; Capozziello et al. 2009). Poplawski (2006) derived a quite complicated expression for the jerk parameter in $f(R)=R-\frac{\alpha^{2}}{3 R}$ (Poplawski 2006):

$$
\begin{aligned}
j^{\prime}= & {\left[\phi f^{\prime}-3 f\right)\left(2 f^{\prime 2}+\phi f^{\prime} f^{\prime \prime}-6 f f^{\prime \prime}\right)\left(30 \phi^{3} f^{\prime 2} f^{\prime \prime 2}\right.} \\
& +10 \phi^{3} f^{\prime 3} f^{\prime \prime \prime}-150 \phi^{2} f f^{\prime} f^{\prime \prime 2}-37 \phi^{2} f^{\prime 3} f^{\prime \prime} \\
& -75 \phi^{2} f f^{\prime 2} f^{\prime \prime \prime}-8 \phi f^{\prime 4}+24 f f^{\prime 3}+189 \phi f^{2} f^{\prime \prime 2} \\
& \left.+189 \phi f^{2} f^{\prime} f^{\prime \prime \prime}+192 \phi f f^{\prime 2} f^{\prime \prime}-162 f^{3} f^{\prime \prime \prime}-267 f^{2} f^{\prime} f^{\prime \prime}\right) \\
& -\left(2 \phi^{2} f^{\prime 4}+10 \phi^{3} f^{\prime 3} f^{\prime \prime}-75 \phi^{2} f^{\prime 2} f f^{\prime \prime}-12 \phi f f^{\prime 3}\right. \\
& \left.+189 \phi f^{2} f^{\prime} f^{\prime \prime}-162 f^{3} f^{\prime \prime}\right) \times\left(3 \phi^{2} f^{\prime} f^{\prime \prime 2}-15 \phi f f^{\prime \prime 2}-8 f^{\prime 3}\right. \\
& +18 f^{2} f^{\prime 2}+27 f f^{\prime} f^{\prime \prime}-\phi f^{\prime 2} f^{\prime \prime}+\phi^{2} f^{\prime 2} f^{\prime \prime \prime}-9 \phi f f^{\prime} f^{\prime \prime \prime} \\
& \left.\left.+18 f^{2} f^{\prime \prime \prime}\right)\right] \times\left[\left(\phi f^{\prime}-3 f\right)^{3}\left(2 f^{\prime 2}+\phi f^{\prime} f^{\prime \prime}-6 f f^{\prime \prime}\right)^{2}\right]^{-1} .
\end{aligned}
$$


The snap parameter in this model is (Poplawski 2007)

$s=j^{\prime} \frac{6 f^{\prime}\left(\phi f^{\prime}-2 f\right)}{\left(2 f^{\prime 2}+\phi f^{\prime} f^{\prime \prime}-6 f f^{\prime \prime}\right)}-j \frac{8 \phi f^{\prime}-15 f}{\phi f^{\prime}-3 f}$.

Poplawski (2007) calculated $q_{0}=-0.67_{-0.03}^{+0.06}, j_{0}=1.01_{-0.21}^{+0.08}$ and $s_{0}=-0.22_{-0.19}^{+0.21}$. These expressions of the jerk and snap parameters are only valid in Palatini variational principle. A generic formula for the cosmographic parameter is derived by Capozziello, Cardone \& Salzano (2008; for more details, see Eqs. (23)-(33) in their paper). They also gave the best fitted value: $q_{0}=-0.55 \pm 0.38, j_{0}=1.0 \pm 5.4$ and $s_{0}=-0.35 \pm 28.1$ using SNe Ia. In this paper, we only use Poplawski (2006) as an example for the $f(R)$ gravity.

\section{Constraints from SNe la and GRBs}

Davis et al. (2007) fitted the SNe Ia dataset that includes 60 ESSENCE SNe Ia (WoodVasey et al. 2007), 57 SNe Ia from Super-Nova Legacy Survey (SNLS) (Astier et al. 2006), 45 nearby SNe Ia and 30 SNe Ia detected by HST (Riess et al. 2007) with the MCLS2K2 method. With the luminosity distance $d_{\mathrm{L}}$ in units of megaparsecs, the predicted distance modulus is

$\mu=5 \log \left(d_{\mathrm{L}}\right)+25$.

The likelihood functions can be determined from the $\chi^{2}$ statistic,

$\chi_{\mathrm{SNe}}^{2}=\sum_{i=1}^{N} \frac{\left[\mu_{i}\left(z_{i}\right)-\mu_{0, i}\right]^{2}}{\sigma_{\mu_{0, i}}^{2}+\sigma_{v}^{2}}$,

where $\sigma_{v}$ is the dispersion in the supernova redshift (transformed to distance modulus) due to peculiar velocities, $\mu_{0, i}$ is the observational distance modulus, and $\sigma_{\mu_{0, i}}$ is the uncertainty in the individual distance moduli. The confidence regions can be found by marginalizing the likelihood functions over $H_{0}$ (i.e., integrating the probability density $p \propto \exp ^{-\chi^{2} / 2}$ for all values of $H_{0}$ ).

We use the calibration results obtained by using the interpolation methods directly from SNe Ia data (Liang et al. 2008). The calibrated luminosity relations are completely cosmology independent. We assume that these relations do not evolve with redshift and are valid in $z>1.40$. The luminosity or energy of GRB can be calculated. Thus, the luminosity distances and distance modulus can be obtained. After obtaining the distance modulus of each burst using one of these relations, we use the same method as Schaefer (2007) to calculate the real distance modulus,

$\mu_{\mathrm{fit}}=\left(\sum_{i} \mu_{i} / \sigma_{\mu_{i}}^{2}\right) /\left(\sum_{i} \sigma_{\mu_{i}}^{-2}\right)$,

where the summation runs from 1-5 over the relations with available data, $\mu_{i}$ is the best estimated distance modulus from the $i$ th relation, and $\sigma_{\mu_{i}}$ is the corresponding uncertainty. The uncertainty of the distance modulus for each burst is

$\sigma_{\mu_{\mathrm{fit}}}=\left(\sum_{i} \sigma_{\mu_{i}}^{-2}\right)^{-1 / 2}$.

The $\chi^{2}$ value is

$\chi_{\mathrm{GRB}}^{2}=\sum_{i=1}^{N} \frac{\left[\mu_{i}\left(z_{i}\right)-\mu_{f i t, i}\right]^{2}}{\sigma_{\mu_{\mathrm{ft}, i}}^{2}}$,

where $\mu_{\mathrm{fit}, i}$ and $\sigma_{\mu_{\mathrm{fit}, i}}$ are the fitted distance modulus and its error.

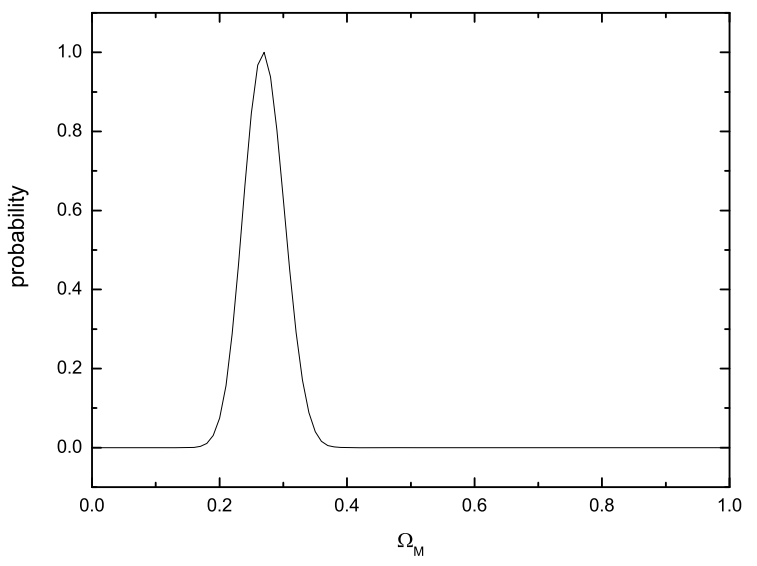

Fig. 1. Luminosity distance-redshift diagram. The circles are the GRBs. The solid line is the result of our fitting.

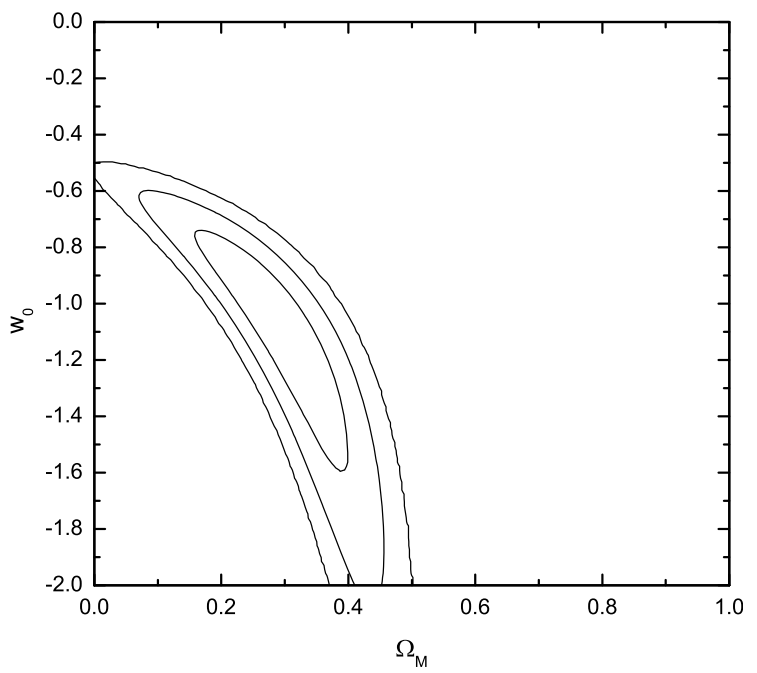

Fig. 2. Constraints on $\Omega_{\mathrm{M}}$ and $w$ from $1 \sigma$ to $3 \sigma$ using $192 \mathrm{SNe}$ Ia in the $w=w_{0}$ model

We combine SNe Ia and GRBs by multiplying the likelihood functions. The total $\chi^{2}$ value is $\chi_{\text {total }}^{2}=\chi_{\mathrm{SNe}}^{2}+\chi_{\mathrm{GRB}}^{2}$. The best fitted value is obtained by minimizing $\chi_{\text {total }}^{2}$.

\subsection{Constraints on cosmographic parameters}

In our analysis, we consider a flat cosmology. We use $h=0.72 \pm$ 0.08 from the Hubble Space Telescope key projects (Freedman et al. 2001).

Let us first consider observational constraints on dark energy models. In Fig. 1, we show the distribution probabilities as a function of $\Omega_{\mathrm{M}}$ in the flat $\Lambda \mathrm{CDM}$ model from SNe Ia and GRBs. From this figure, we have $\Omega_{\mathrm{M}}=0.27 \pm 0.04$. The cosmographic parameters in $\Lambda \mathrm{CDM}$ model are $q_{0}=-1+\frac{3}{2} \Omega_{\mathrm{M}}, j_{0}=1.0$ and $s_{0}=1-\frac{9}{2} \Omega_{\mathrm{M}}$. We can obtain $q_{0}=-0.60 \pm 0.06, j_{0}=1.0$ and $s_{0}=-0.22 \pm 0.18$.

In Fig. 2 we present constraints on $\Omega_{\mathrm{M}}$ and $w$ from $1 \sigma$ to $3 \sigma$ using $192 \mathrm{SNe}$ Ia and $69 \mathrm{GRBs}$ in the $w=w_{0}$ model. We measure $\Omega_{\mathrm{M}}=0.29_{-0.14}^{+0.11}$ and $w_{0}=-1.04_{-0.52}^{+0.32}$. The cosmographic parameters in the $w=w_{0}$ model are $q_{0}=-0.61_{-0.60}^{+0.38}, j_{0}=1.13_{-1.79}^{+1.10}$ and $s_{0}=-0.08_{-2.75}^{+1.75}$.

In Fig. 3 we present constraints on $w_{0}$ and $w_{1}$ from $1 \sigma$ to $3 \sigma$ using $192 \mathrm{SNe}$ Ia and $69 \mathrm{GRBs}$ in the $w=w_{0}+w_{1} z$ model. The values of the parameters are 


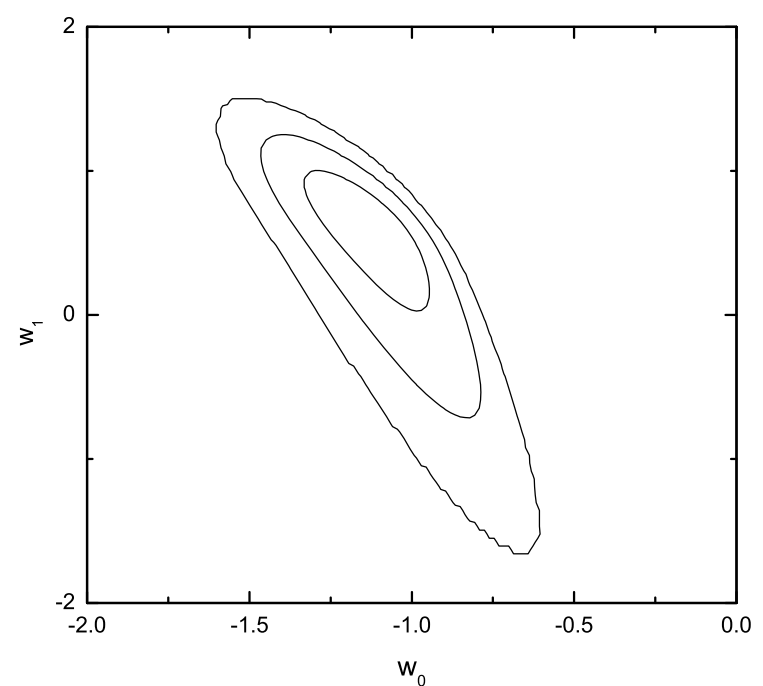

Fig. 3. The same as Fig. 2 but in the $w=w_{0}+w_{1} z$ model.

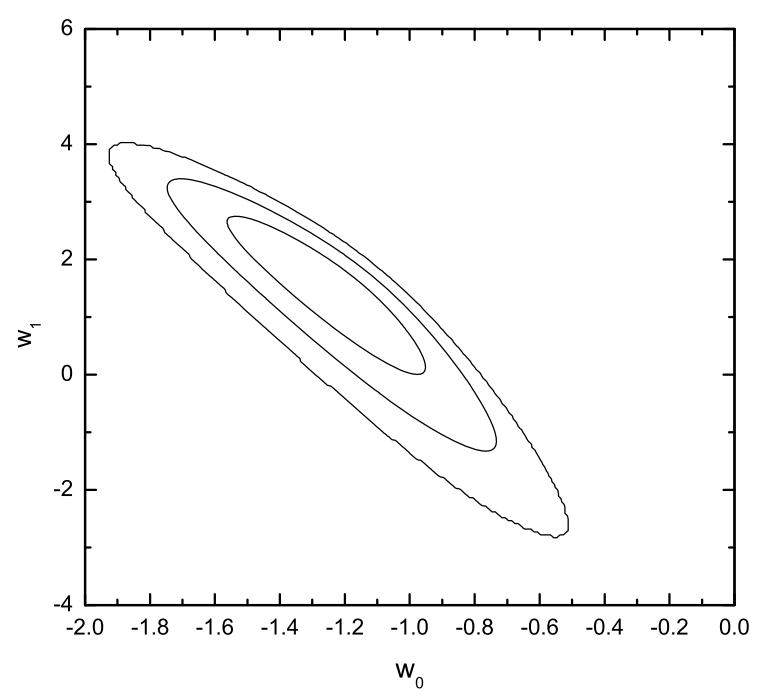

Fig. 4. The same as Fig. 2 but in the $w=w_{0}+w_{1} z /(1+z)$ model.

$w_{0}=-1.14 \pm 0.19$ and $w_{1}=0.63 \pm 0.44$. The cosmographic parameters are $q_{0}=-0.75 \pm 0.21, j_{0}=2.21 \pm 0.93$ and $s_{0}=-12.25 \pm 9.18$.

In Fig. 4 we present constraints on $w_{0}$ and $w_{1}$ from $1 \sigma$ to $3 \sigma$ using 192 SNe Ia and 69 GRBs in the $w=w_{0}+w_{1} z /(1+z)$ model. We measure $w_{0}=-1.22 \pm 0.30$ and $w_{1}=1.6_{-1.10}^{+1.20}$. The cosmographic parameters are $q_{0}=-0.90 \pm 0.33, j_{0}=3.93_{-2.09}^{+1.93}$ and $s_{0}=-25.52_{-25.33}^{+27.33}$.

Figure 5 shows constraints on $A_{s}$ and $\alpha$ from $1 \sigma$ to $3 \sigma$ using SNe Ia and GRBs in the GCG model. The parameters are $A_{s}=$ $0.79 \pm 0.12$ and $\alpha=0.25_{-0.75}^{+0.95}$. The cosmographic parameters are $q_{0}=-0.695 \pm 0.18, j_{0}=1.18_{-0.65}^{+0.78}$ and $s_{0}=-0.37_{-1.48}^{+1.85}$.

In Fig. 6 we present constraints on $\Omega_{\mathrm{M}}$ and $n$ from $1 \sigma$ to $3 \sigma$ using 192 SNe Ia and 69 GRBs in the Cardassian expansion model. We measure $\Omega_{\mathrm{M}}=0.29 \pm 0.11$ and $n=-0.07_{-0.46}^{+0.34}$. The cosmographic parameters are $q_{0}=-0.67_{-0.40}^{+0.35}, j_{0}=1.35_{-1.45}^{+1.20}$ and $s_{0}=0.36_{-2.85}^{+2.51}$.

Figure 7 shows constraints on $\Omega_{\mathrm{M}}$ using SNe Ia and GRBs in the DGP model. The value of $\Omega_{M}$ is $\Omega_{M}=0.20 \pm 0.02$. The cosmographic parameters are $q_{0}=-0.50 \pm 0.04, j_{0}=0.83 \pm 0.02$ and $s_{0}=-0.56 \pm 0.12$.

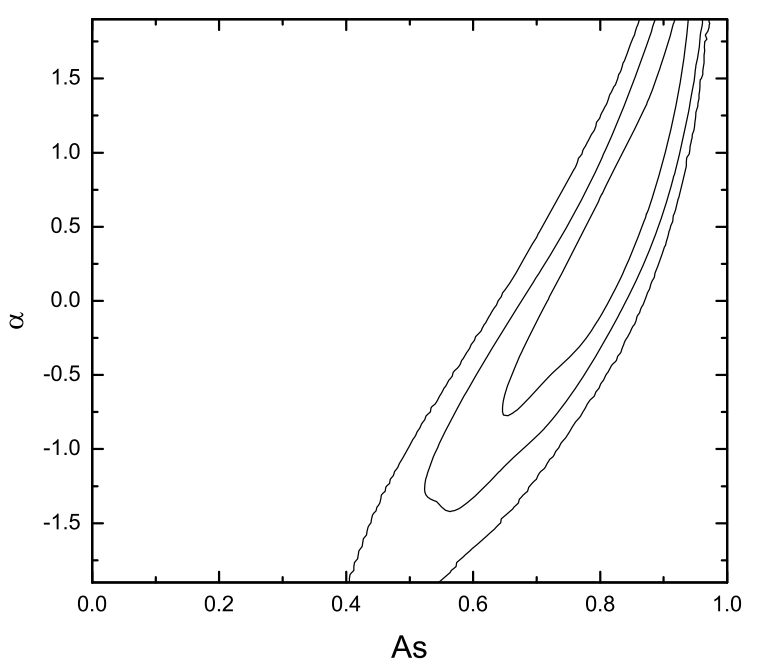

Fig. 5. The same as Fig. 2 but in the GCG model.

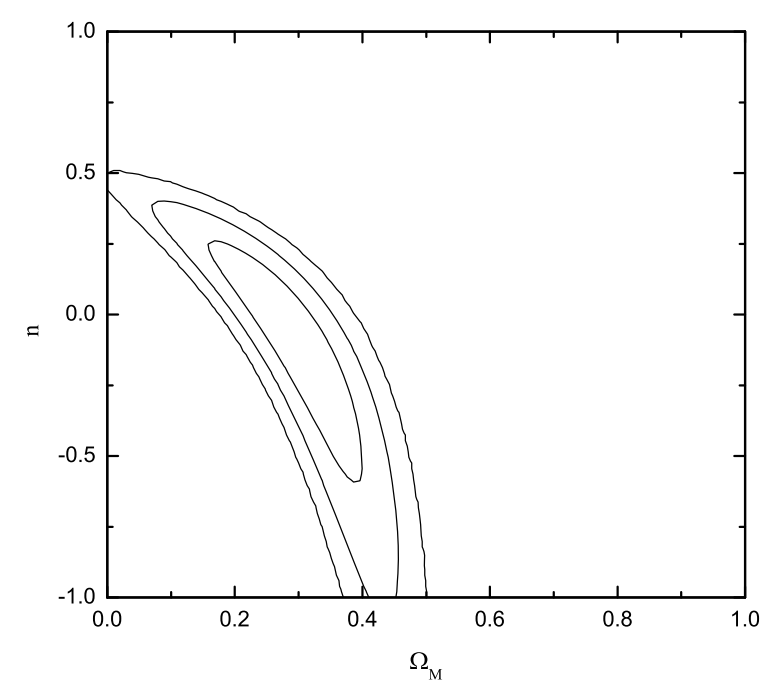

Fig. 6. The same as Fig. 2 but in the Cardassian expansion model.

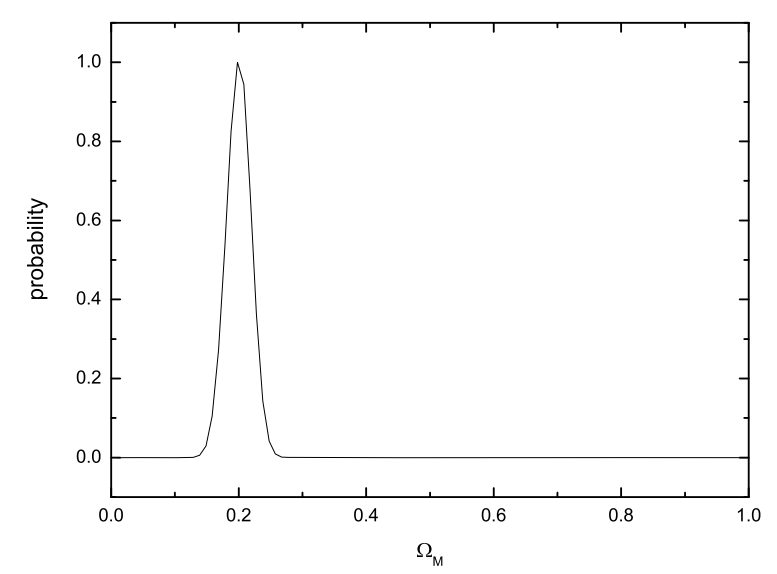

Fig. 7. The same as Fig. 1 but in the DGP model.

We directly use Eq. (5) to constrain the cosmographic parameters. This analysis uses the FRW metric only, so we have not specified any gravitational theory yet. The luminosity distance only depends on redshift $z$ and cosmographic parameters. So this method is fully model independent. We use the $192 \mathrm{SNe}$ Ia and $69 \mathrm{GRBs}$ and find the best fit parameters are $q_{0}=-0.85 \pm 0.19$, 
Table 1. The cosmographic parameter values.

\begin{tabular}{cccc}
\hline \hline Model & $q_{0}$ & $j_{0}$ & $s_{0}$ \\
\hline$\Lambda$ CDM & $-0.60 \pm 0.06$ & $1.0 \pm 0.11$ & $-0.22 \pm 0.18$ \\
$w=w_{0}$ & $-0.61_{-0.60}^{+0.38}$ & $1.13_{-1.79}^{+1.10}$ & $-0.08_{-2.75}^{+1.75}$ \\
$w=w_{0}+w_{1} z$ & $-0.75 \pm 0.21$ & $2.21 \pm 0.93$ & $-12.25 \pm 9.18$ \\
$w=w_{0}+\frac{w_{1} z}{1+z}$ & $-0.90 \pm 0.33$ & $3.93_{-2.90}^{+1.93}$ & $-25.52_{-25.33}^{+27.33}$ \\
GCG & $-0.70 \pm 0.18$ & $1.18_{-0.65}^{+0.78}$ & $-0.37_{-1.48}^{+1.35}$ \\
Cardassian & $-0.67_{-0.40}^{+0.35}$ & $1.20_{-1.45}^{+1.20}$ & $0.22_{-2.85}^{+2.51}$ \\
DGP & $-0.50 \pm 0.04$ & $0.83 \pm 0.02$ & $-0.56 \pm 0.12$ \\
$f(R)$ & $-0.67_{-0.03}^{+0.06}$ & $1.01_{-0.21}^{+0.08}$ & $-0.22_{-0.19}^{+0.21}$ \\
\hline
\end{tabular}

$j_{0}=1.50 \pm 0.80$ and $s_{0}=3.49 \pm 4.50$. The results are consistent with the flat $\Lambda \mathrm{CDM}$ model.

In Table 1 we summarize the constraints on cosmographic parameters. The deceleration and jerk parameters in the $w=w_{0}$, GCG, Cardassian expansion and $f(R)$ models are almost the same in the $1 \sigma$ confidence level. These values are consistent with the deceleration and jerk parameters of the $\Lambda \mathrm{CDM}$ model in the $1 \sigma$ confidence level. So these models cannot be discriminated between by using the present value of the statefinder pair. However the snap parameter in all the models is different and thus can be used to discriminate between the cosmological models. In the future, more data will give a precise snap parameter in different models.

\section{Discussions and conclusions}

The cosmic acceleration could be due to unidentified dark energy, or a modification of general relativity (modified gravity). In this paper we investigate the deceleration, jerk and snap parameters in modified gravity and dark energy models. We calibrated the GRB luminosity relations using $\mathrm{SNe}$ Ia without assuming any cosmological models. Because gamma-ray bursts can be detected at high redshifts, we calculated the deceleration, jerk and snap parameters using type Ia supernovae and gamma-ray bursts. GRBs can extend the redshift-distance relation up to high redshifts. We find that the deceleration and jerk parameters in the $w=w_{0}, \mathrm{GCG}$, Cardassian expansion and $f(R)$ models are almost the same at a $1 \sigma$ confidence level. So these models cannot be discriminated between using the present value of the statefinder pair. We found that the dark energy models and modified gravity models could be distinguished between by the snap parameter. Using the model-independent constraints on cosmographic parameters, we found that the $\Lambda \mathrm{CDM}$ model is consistent with the current data.

Acknowledgements. This work is supported by the National Natural Science Foundation of China (grants 10233010, 10221001 and 10873009) and the National Basic Research Program of China (973 program) No. 2007CB815404. F. Y. Wang was also supported by the Jiangsu Project Innovation for Ph.D. Candidates (CX07B-039z).

\section{References}

Alam, U., Sahni, V., Deep Saini, T., \& Starobinsky, A. A. 2003, MNRAS, 344, 1057

Allen, S. W., Schmidt, R. W., Ebeling, H., Fabian, A. C., \& van Speybroeck, L. 2004, MNRAS, 353, 457
Allen, S. W., Rapetti, D. A., Schmidt, R. W., et al. 2008, MNRAS, 383, 879 Astier, P., Guy, J., Regnault, N., et al. 2006, A\&A, 447, 31 Bertolami, O., \& Silva, P. T. 2006, MNRAS, 356, 1149 Basilakos, S., \& Perivolaropoulos, L. 2008, MNRAS, 391, 411 Bennett, C. L., Hill, R. S., Hinshaw, G., et al. 2003, ApJS, 148, 97 Bento, M. C., Bertolami, O., \& Sen, A. A. 2002, Phys. Rev. D, 66, 043507 Caldwell, R. R., \& Kamionkowski, M. 2004, JCAP, 0409, 009 Caldwell, R. R., Dave, R., \& Steinhardt, P. J. 1998, Phys. Rev. Lett., 80, 1582 Capozziello, S., \& Izzo, L. 2008, A\&A, 490, 31

Capozziello, S., Cardone, V. F., \& Salzano, V. 2008, Phys. Rev. D, 78, 063504 Capozziello, S., Elizalde, E., Nojiri, S., \& Odintsov, S. D. 2009, Phys. Lett. B, 671,193

Cardone, V. F., Capozziello, S., \& Dainotti, M. G. 2009, [arXiv:0901. 3194]

Carroll, S. M., et al. 2004, Phys. Rev. D, 70, 043528

Cattoën, C, \& Visser, M. 2007, [gr-qc/0703122v3]

Chevallier, M., \& Polarski, D. 2001, Int. J. Mod. Phys. D, 10, 213

Dai, Z. G., Liang, E. W., \& Xu, D. 2004, ApJ, 612, L101

Davis, T. M., Mörtsell, E., Sollerman, J., et al. 2007, ApJ, 666, 716

Deffayet, C., Dvali, G. R., \& Gabadadze, G. 2002, Phys. Rev. D, 65, 044023

Ciardi, B., \& Loeb, A. 2000, ApJ, 540, 687

Dvali, G. R., Gabadadze, G., \& Porrati, M. 2000, Phys. Lett. B, 485, 208

Di Girolamo, T., et al. 2005, JCAP, 4, 008

Eisenstein, D. J., Zehavi, I., Hogg, D. W., et al. 2005, ApJ, 633, 560

Firmani, C., Ghisellini, G., Ghirlanda, G., \& Avila-Reese, V. 2005, MNRAS, 360, L1

Fenimore, E. E., \& Ramirez-Ruiz, E. 2000, [arXiv:astro-ph/0004176]

Freedman, W. L., Madore, B. F., Gibson, B. K., et al. 2001, ApJ, 553, 47

Freese, K., \& Lewis, M. 2002, Phys. Lett. B, 540, 1

Friedmann, A. S., \& Bloom, J. S. 2005, ApJ, 627, 1

Ghirlanda, G., Ghisellini, G., Lazzati, D., \& Firmani, C. 2004, ApJ, 613, L13

Gorini, V., Kamenshchik, A., \& Moschella, U. 2003, Phys. Rev. D, 67, 063509

Huterer, D., \& Linder, E. V. 2007, Phys. Rev. D, 75, 023519

Ishak, M., Upadhye, A., \& Spergel, D. N. 2006, Phys. Rev. D, 74, 043513

Kamenshchik, A., Moschella, U., \& Pasquier, V. 2001, Phys. Lett. B, 511, 265

Kodama, Y., Yonetoku, D., Murakami, T., et al. 2008, MNRAS, 391, L1

Kunz, M., \& Sapone, D. 2007, Phys. Rev. Lett., 98, 121301

Lamb, D. Q., Ricker, G. R., Lazzati, D., et al. 2005, [arXiv: astro-ph/0507362]

Li, H., Su, M., Fan, Z., Dai, Z., \& Zhang, X. 2008, Phys. Lett. B, 658, 95

Liang, E. W., \& Zhang, B. 2005, ApJ, 633, 611

Liang, E. W., \& Zhang, B. 2006, MNRAS, 369, L37

Liang, N., Ke Xiao, W., Liu, Y., \& Zhang, N. S. 2008, ApJ, 685, 354

Linder, E. V. 2003, Phys. Rev. Lett., 90, 091301

Linder, E. V. 2005, Phys. Rev. D, 72, 043529

Maor, I., Brustein, R., \& Steinhardt, P. J. 2001, Phys. Rev. Lett., 87, 049901

Peebles, P. J. E., \& Ratra, B. 1988, ApJ, 325, L17

Perlmutter, S., Aldering, G., Goldhaber, G., et al. 1999, ApJ, 517, 565

Polarski, D., \& Gannouji, R. 2008, Phys. Lett. B, 660, 439

Poplawski, N. J. 2006, Phys. Lett. B, 640, 135

Poplawski, N. J. 2007, Class. Quantum. Grav., 24, 3013

Qi, S., Wang, F. Y., \& Lu, T. 2008a, A\&A, 483, 49

Qi, S., Wang, F. Y., \& Lu, T. 2008b, A\&A, 487, 853

Olivares, F., Kruehler, T., Greiner, J., \& Filgas, R. 2009, GCN, 9215

Rapetti, D., Allen, S. W., Amin, M. A., \& Blandford, R. D. 2007, MNRAS, 375, 1510

Riess, A. G., Filippenko, A. V., Challis, P., et al. 1998, AJ, 116, 1009

Riess, A. G., Strolger, L.-G., Tonry, J., et al. 2004, ApJ, 607, 665

Riess, A. G., Strolger, L.-G., Casertano, S., et al. 2007, ApJ, 659, 98

Sahni, V., Saini, T. D., Starobinsky, A. A., \& Alam, U. 2003, JETP Lett., 77, 201

Schaefer, B. E. 2007, ApJ, 660, 16

Silva, P. T., \& Bertolami, O. 2003, ApJ, 599, 829

Spergel, D. N., Verde, L., Peiris, H. V., et al. 2003, ApJS, 148, 175

Spergel, D. N., Bean, R., Doré, O., et al. 2007, ApJS, 170, 377

Tegmark, M., et al. 2006, Phys. Rev. D, 74, 123507

Visser, M. 2004, Class. Quant. Grav., 21, 2603

Vollick, D. N. 2003, Phys. Rev. D, 68, 063510

Wang, F. Y., \& Dai, Z. G. 2006, MNRAS, 368, 371

Wang, F. Y., Dai, Z. G., \& Zhu, Z. H. 2007, ApJ, 667, 1

Wang, F. Y., Dai, Z. G., \& Qi, S. 2009, RAA, 9, 547

Wang, Y., Freese, K., Gondolo, P., \& Lewis, M. 2003, ApJ, 594, 25

Weinberg, S. 1989, Rev. Mod. Phys., 61, 1

Weller, J., \& Albrecht, A. 2001, Phys. Rev. Lett., 86, 1939

Wood-Vasey, W. M., Miknaitis, G., Stubbs, C. W., et al. 2007, ApJ, 666, 694

Wright, E. L. 2007, ApJ, 664, 633

Xu, D., Dai, Z. G., \& Liang. E. W. 2005, ApJ, 633, 603 